\title{
Injuria cerebral aguda causada por oclusión de gran vaso por COVID-19
}

\author{
Acute brain injury caused by occlusion of large vessel by COVID - 19
}

Santiago Gerardo Ruiz-Laos ${ }^{1, a *}$, Aníbal Valentín Díaz-Lazo $2, b$

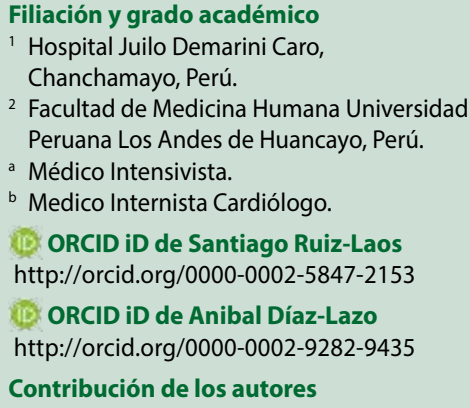

2 Facultad de Medicina Humana Universidad

Peruana Los Andes de Huancayo, Perú.

Médico Intensivista.

${ }^{b}$ Medico Internista Cardiólogo.

Q. ORCID iD de Santiago Ruiz-Laos

http://orcid.org/0000-0002-5847-2153

(1) ORCID iD de Anibal Díaz-Lazo http://orcid.org/0000-0002-9282-9435

Contribución de los autores

SRL: diseño del estudio, recolección de los datos, análisis e interpretación de los datos, en la redacción del manuscrito y aprobó la versión a publicar.

ADL: participó en el diseño del estudio, análisis e interpretación de los datos, en la redacción del manuscrito y aprobó la versión a publicar.

Fuentes de financiamiento

Autofinanciado.

Conflictos de interés

ADL: forma parte del equipo editorial de la Revista Peruana de Ciencias de la Salud, reservándose de participar en el proceso de revisión.

Recibido: 07-04-2020

Arbitrado por pares

Aceptado: 23-05-2020

Citar como

Ruiz-Laos S, Díaz-Lazo A. Injuria cerebral aguda causada por oclusión de gran vaso por COVID-19. Rev Peru Cienc Salud. 2020; 2(2): 109-13. doi: https://doi.org/10.37711/rpcs.2020.2.2.125

Correspondencia

Santiago Gerardo Ruiz-Laos.

Dirección: Av. Chacra Grande parcel 18

manzana E Lote 11. Carabayllo. Lima- Perú.

Código Postal: 15122.

Cel.: 954862003

Email: drgerul@hotmail.com

\section{RESUMEN}

En la pandemia de la enfermedad por la COVID-19 causada por el virus SARS-CoV2, la mayoría de los pacientes presentan síntomas generales y respiratorios, algunos cuadros atípicos gastrointestinales, cardiovasculares, dermatológicos o neurológicos. Se reporta el caso de un paciente varón de 40 años, sin ningún antecedente epidemiológico importante, diagnosticado de COVID 19 por prueba rápida inmunológica, quien presenta un accidente cerebro vascular isquémico súbito. En el estudio tomográfico cerebral sin contraste se evidenció un área extensa isquémica, en el hemisferio cerebral izquierdo en territorio de la arteria cerebral media, acompañado de edema. En los pacientes con COVID-19, infectados por coronavirus SARS-CoV2, debe tenerse en cuenta la posibilidad de que el paciente presente accidente cerebrovascular (ACV), para permitir un manejo adecuado y oportuno.

Palabras clave: coronavirus, COVID-19, Pandemias, Arteria cerebral media, Isquemia cerebral, síndrome respiratorio agudo severo, infecciones, Virus del SARS, Cerebro, Edema, Tomografía, rayos $X$ computados Cerebro, Pruebas inmunologicas (Fuente: DeCS-BIREME).

\section{ABSTRACT}

In the 2019 coronavirus disease pandemic (COVID-19) caused by the SARS-CoV2 virus, the majority of patients present general and respiratory symptoms, some atypical gastrointestinal, cardiovascular, dermatological or neurological symptoms. In the case report of a 40-year-old male patient, without any important epidemiological history, diagnosed with COVID-19 by rapid immunological test, who presents with a sudden ischemic stroke. In the CT scan of the brain without contrast, a large ischemic area is evident in the left cerebral hemisphere in the middle cerebral artery territory, accompanied by edema. In patients with COVID-19, infected with the SARSCoV2 coronavirus, the possibility of the patient having a cerebrovascular accident (CVA) should be taken into account, to allow adequate and timely management.

Keywords: coronavirus, COVID-19, Pandemics, Middle Cerebral Artery, Brain Ischemia, severe acute respiratory syndrome, Infections, SARS Virus, Brain, Edema, Tomography, X-Ray Computed Cerebrum, Immunologic Tests (Source: MeSH-NLM). 


\section{INTRODUCCIÓN}

La repentina e inesperada interrupción del flujo sanguíneo hacía el cerebro, es denominado como accidente cerebrovascular (ACV), y se define como injuria encefálica aguda al síndrome clínico que se presenta en forma aguda, que va acompañado de edema cerebral y depresión funcional neurológica, debido a una lesión en el encéfalo, la cual puede ser reversible o no. El ACV isquémico agudo causado por una oclusión de grandes vasos se asocia con una frecuencia de mortalidad del $80 \%$ de los $\operatorname{casos}^{(1)}$. El ACV puede ser de origen isquémico o hemorrágico, siendo la principal causa de discapacidad y la cuarta causa de muerte en países desarrollados ${ }^{(2)}$. $\mathrm{Si}$ bien existe un incremento en la incidencia y prevalencia de esta enfermedad, el uso de programas de formación y la optimización del tratamiento agudo han demostrado ser eficaces en la reducción de morbimortalidad en los últimos años ${ }^{(3,4)}$.

Algunas investigaciones apuntan a que los ACV en pacientes infectados con la enfermedad del coronavirus (COVID-19), podrían ser consecuencia directa del mismo trastorno en coagulación, en la producción de trombos, medido por el predictor negativo del dímero $D(D D)^{(5)}$. Por lo tanto, aquellos que se forman en las paredes de los vasos de las piernas, por ejemplo, pueden migrar hasta los pulmones, causando un bloqueo conocido como tromboembolia pulmonar, responsable de la falla respiratoria aguda y severa, en muchos pacientes con COVID-19. Los trombos formados dentro o cerca del corazón conducirían a un síndrome coronario agudo (SICA), otra complicación en pacientes con COVID-19, y por último, los coágulos que se formen más arriba terminarán en los vasos cerebrales, ocasionando el $A C V{ }^{(6)}$. Stevens, intensivista del Hospital John Hopkins de Baltimore, menciona que estos ACV son "una de las manifestaciones más dramáticas" de los trastornos de coagulación de la sangre en pacientes con COVID-19 y están presentando la forma más letal de $\mathrm{ACV}{ }^{(7)}$. A continuación, se reporta un caso de un paciente adulto, relativamente joven, sin ningún factor de riesgo con COVID-19, que presentó ACV causado por la oclusión de una arteria de gran calibre, atípica en su presentación, y se realiza, al hilo del mismo, la revisión de la literatura.

\section{REPORTE DE CASO}

Paciente varón de 40 años de edad, natural de Lima, infante de la Marina de Guerra del Perú, quien realiza actividad física en forma regular durante la semana y quien no presenta ningún antecedente importante desde el punto de vista médico (sus familiares niegan hipertensión arterial, diabetes mellitus y tuberculosis pulmonar), quien ingresa a la unidad de trauma shock del servicio de emergencia del Hospital Regional de Huacho, traído por sus familiares, por presentar un cuadro caracterizado por trastorno del sensorio, dificultad para hablar, inestabilidad para ponerse de pie y tendencia al sueño; con un periodo de la enfermedad de aproximadamente 12 horas de evolución, el cual cursa en forma insidiosa y progresiva. Debido al aparente mal estado general, cursan interconsulta al intensivista de turno para la evaluación respectiva.

Al realizarle el examen de funciones vitales, se evidencia que estas se encuentran dentro de valores y rangos normales con frecuencia cardiaca de 78 ppm, frecuencia respiratoria 18 respiraciones por minuto, temperatura $36{ }^{\circ} \mathrm{C}$, saturación $98 \%$ a $\mathrm{FiO}_{2} 21 \%$; sin embargo, los valores de la presión arterial se encontraban incrementados, con un valor de $140 / 85 \mathrm{mmHg}$. Al examen de la evaluación médica por aparatos y sistemas, se observa el siguiente cuadro: aparato cardiovascular (ruidos cardiacos rítmicos, bajo tono, no soplos); aparato respiratorio (murmullo vesicular conservado en ambos campos pulmonares con presencia de leves crépitos en ambas bases pulmonares); examen de abdomen (blando depresible, ruidos hidro aéreos presentes, no masas palpables); y a la evaluación neurológica (se evidencia escala de Glasgow 9/15 puntos, [apertura ocular 2 puntos, respuesta verbal 2 puntos y respuesta motora 5 puntos], conjuntamente con un estado somnoliento, pupilas isocóricas en $3 / 4 \mathrm{~mm}$, hipo reactivas y con hemiplejia derecha). En exámenes de laboratorio se evidencia hemograma con leve leucocitosis y la bioquímica con valores normales (ver tabla 1). Además, se realiza la prueba rápida, para descarte de infección por COVID-19, siendo el resultado de dicha prueba positivo, tanto para IgM e lgG. De igual forma, al realizar el examen de $\mathrm{DD}$, este fue positivo.

Por otro lado, al visualizar la placa de la radiología torácica, se evidencia una silueta cardíaca dentro de lo normal, con un índice cardiaco dentro de rangos normales, y parénquima pulmonar dentro de lo normal. Al paciente se le realiza estudio tomográfico espiral multicorte, sin contraste, en donde se evidencia una extensa área isquémica cortico sub cortical en el parénquima hemi cerebral izquierdo, en zona témporo-parieto-occipital, en territorio de la arteria cerebral media (ver figura 1).

Por lo tanto, con la evaluación correspondiente y en la realización de la unidad clínica se concluyó los siguientes diagnósticos: (1) accidente cerebrovascular isquémico por COVID-19, (2) encefalopatía, (3) edema cerebral, (4) hipertensión intracraneal, (5) D/C neumonía aspirativa. 
Tabla 1. Valores laboratoriales al ingreso

\begin{tabular}{|c|c|c|c|}
\hline $\begin{array}{l}\text { Examen de } \\
\text { laboratorio }\end{array}$ & $\begin{array}{c}\text { Valor } \\
\text { encontrado }\end{array}$ & Unidades & Rango referencial \\
\hline \multicolumn{4}{|l|}{ Hemograma } \\
\hline $\begin{array}{l}\text { Glóbulos } \\
\text { blancos }\end{array}$ & 13,730 & $\mathrm{~mm} 3$ & $4,000-10,000$ \\
\hline Abastonados \% & 0 & $\%$ & $0,0-5,0$ \\
\hline Hemoglobina & 16,2 & $\mathrm{gr} / \mathrm{dL}$ & $13,5-18,5$ \\
\hline Hematocrito & 50,6 & $\mathrm{gr} / \mathrm{dL}$ & $37-47$ \\
\hline Plaquetas & 335,000 & $\mathrm{~mm} 3$ & $150,000-450,000$ \\
\hline $\ln r$ & 0,87 & $\mathrm{~S} / \mathrm{U}$ & $0,8-1,10$ \\
\hline \multicolumn{4}{|l|}{ Bioquímico } \\
\hline $\begin{array}{l}\text { Fosfatasa } \\
\text { alcalina }\end{array}$ & 101 & $U / I$ & $53,00-128,00$ \\
\hline Urea & 29 & $\mathrm{mg} / \mathrm{dL}$ & $15-39$ \\
\hline TGP & 54 & $U / L$ & $<42$ \\
\hline TGO & 22 & $U / L$ & $<37$ \\
\hline Glucosa & 119 & $\mathrm{mg} / \mathrm{dL}$ & $70-110$ \\
\hline Creatinina & 0,57 & $\mathrm{mg} / \mathrm{dL}$ & $0,9-1,3$ \\
\hline Albumina & 4,68 & $\mathrm{mg} / \mathrm{dL}$ & $3,5-5,5$ \\
\hline Globulina & 2,77 & $\mathrm{mg} / \mathrm{dL}$ & $1,5-3,0$ \\
\hline $\begin{array}{l}\text { Proteínas } \\
\text { totales }\end{array}$ & 7,45 & $\mathrm{mg} / \mathrm{dL}$ & $6,0-8,0$ \\
\hline $\begin{array}{l}\text { Bilirrubina } \\
\text { total }\end{array}$ & 0,77 & $\mathrm{mg} / \mathrm{dL}$ & $<1,1$ \\
\hline \multicolumn{4}{|l|}{ Electrolitos } \\
\hline Sodio & 137 & $\mathrm{mmol} / \mathrm{L}$ & $135-145$ \\
\hline Potasio & 4,0 & $\mathrm{mmol} / \mathrm{L}$ & $3,5-5,5$ \\
\hline Cloro & 103 & $\mathrm{mmol} / \mathrm{L}$ & $95-108$ \\
\hline Dímero d & 1,41 & UGI/L & $<0,5$ \\
\hline
\end{tabular}

Se inicia tratamiento en la unidad de trauma shock, con: (1) reposo absoluto, (2) NPO, (3) CINa 90/000 + CIK $20 \% \mathrm{EV}$ a $80 \mathrm{cc} / \mathrm{h}$, (4) manitol al $20 \% \mathrm{EV}$ a $150 \mathrm{cc} \mathrm{c} / 4 \mathrm{~h}$,

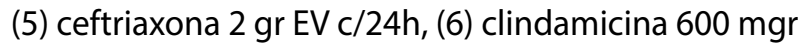
$\mathrm{EV} \mathrm{c} / 6 \mathrm{~h}$, (7) fenitoina $100 \mathrm{mgr} \mathrm{EV} \mathrm{c/8h,} \mathrm{(8)} \mathrm{paracetamol}$ $1 \mathrm{gr}$ por SNG c/8h, (9) ácido acetil salicílico $100 \mathrm{mgr}$ por SNG c/24h, (10) torvastatina 80 mgr por SNG c/24h, (11) clopidroguel $300 \mathrm{mg}$ por SNG c/24h, (12) enoxaparina $60 \mathrm{mgr}$ SC c/24h, (13) azitromicina 500mg por SNG $\mathrm{c} / 24 \mathrm{~h}$, (14) hidroxicloroquina $200 \mathrm{mgr}$ por SNG c/12h, (15) ivermectina 80 gotas por SNG c/24h, (16) omeprazol $40 \mathrm{mgr} \mathrm{EV} \mathrm{c/} \mathrm{24h,} \mathrm{(17)} \mathrm{metoclopramida} 10 \mathrm{mgr} \mathrm{EV} \mathrm{c/8h,}$ (18) hemoglucotes $\mathrm{C} / 8$ horas, (19) diazepam $10 \mathrm{mgr} \mathrm{EV}$ PRN si presenta convulsiones, (20) BHE.

Debido a no contar con camas disponibles en la unidad de cuidados intensivos, el paciente es tratado en la unidad de trauma shock, hasta su referencia a otro hospital de la ciudad de Lima, por el alto riesgo que pueda presentar el paciente por el diagnóstico y la presencia de un incremento de edema intraparenquimal cerebral que haga requerir de craneotomía descompresiva si no hace efecto la terapia de neuroprotección-instauradatranscurridas seis horas de su ingreso, presentando una evolución estacionaria y manteniendo el tratamiento inicial de cobertura antibiótica, neuro protección y soporte, el paciente es referido a un hospital de mayor complejidad.

\section{DISCUSIÓN}

En la actualidad, el mundo está frente al desarrollo de una nueva enfermedad, producida por un virus altamente patógeno y letal, que se describió por primera vez el 31 de diciembre del pasado año, en Wuhan (provincia de Hubei, China). La enfermedad fue nombrada coronavirus disease 2019 (COVID-19) y el 11 de marzo de 2020 la Organización Mundial de la Salud la consideró una pandemia ${ }^{(8,9)}$.

Durante las últimas décadas, la investigación en medicina ha demostrado que la mayoría de los coronavirus humanos se presentan inicialmente con enfermedades respiratorias leves, sin embargo, el SARS-CoV, elMERS-CoV y más recientemente la COVID-19, presentan sintomatología muy similar con una elevada mortalidad ${ }^{(10)}$. Por lo tanto, ya se ha demostrado que los pacientes infectados con COVID-19 pueden presentarse con diversa sintomatología, incluso con síntomas neurológicos, y que estos pueden presentarse antes, durante o después de síntomas pulmonares y fiebre ${ }^{(11)}$.

A la fecha, existe muy poca información relacionada a las manifestaciones neurológicas de la viremia por COVID-19, sin embargo, se menciona que hasta cerca de un $40 \%$ de los pacientes hospitalizados en Wuhan con COVID-19, manifestaron sintomatología neurológica ${ }^{(12)}$, como es el caso de nuestro paciente.

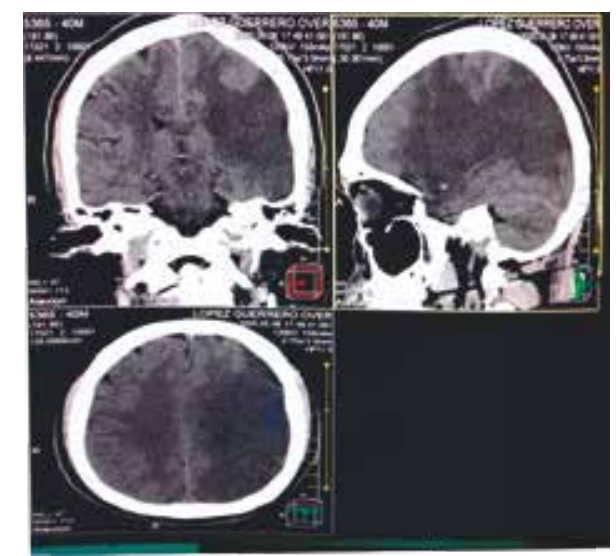

Figura 1. Tomografía de ingreso. Se aprecia el encéfalo en los planos coronal, sagital y trasversal; evidenciándose área isquémica extensa en territorio de la arteria cerebral media, con edema cerebral difuso 
De los pacientes mencionados, se observó que las manifestaciones neurológicas encontradas fueron: mareos $(16,8 \%)$, cefalea $(13,1 \%)$ y encefalopatía $(2,8 \%)$. Los signos y síntomas periféricos más comunes fueron: anosmia (5,1\%), disgeusia (5,6 \%) y lesión muscular (10,1\%). El accidente cerebrovascular, el ACV complicó la estancia hospitalaria por COVID-19 en el 5,9\% de los pacientes, con una mediana de 10 días después del inicio de los síntomas. Los pacientes con ACV eran, por lo general, adultos mayores (mayores de 70 años de edad), tenían más comorbilidades cardiovasculares, y la neumonía fue más grave. Los mecanismos fisiopatológicos encontrados del $A C V$, según dichos autores, fueron la hipercoagulabilidad por enfermedad crítica y cardioembolismo relacionado con el virus ${ }^{(13)}$. Sin embargo, nuestro paciente no presentó ningún signo periférico y, lo que es aún más llamativo, la edad a la cual se presentó este evento es casi a la mitad de la edad promedio de los pacientes en donde se presentó esta injuria cerebral. Por otro lado, el paciente, motivo de estudio, presentó valores dentro de lo normal de plaquetas. Con lo cual, la presentación de la infección por COVID-19 manifiesta una presentación distinta.

En el estudio realizado por Hai-Yang ${ }^{(14)}$ reporta que pacientes diagnosticados con COVID-19 solo presentaron sintomatología neurológica relacionada a: (1) manifestaciones inespecíficas como cefalea, languidez, marcha inestable y malestar general; (2) hemorragia cerebral; (3) infarto cerebral, y 4) otras enfermedades neurológicas previamente diagnosticadas como de base.

Recientemente en la publicación de casos clínicos, se están evidenciando que las afectaciones neurológicas en infecciones graves empeoran el pronóstico de los pacientes ${ }^{(15)}$. El estudio del potencial neurotropo del virus y su aislamiento del encéfalo (del endotelio de la microcirculación cerebral, del líquido cefalorraquídeo y parénquima encefálico), demuestra un papel esencial en el daño cerebral. Los informes de autopsias han revelado edema del tejido cerebral y degeneración neuronal parcial en pacientes fallecidos ${ }^{(16,17)}$. En el caso del paciente de estudio, la extensa área del infarto cerebral sumada al daño que ocasiona la infección por COVID-19 a nivel cerebral, podría contribuir a un pronóstico desfavorable.

Así mismo se ha comprobado que los pacientes con infecciones graves por SARS-CoV-2, muestran valores séricos de $\mathrm{DD}$ altos y disminución del nivel de plaquetas ${ }^{(18)}$. Por lo tanto, se ha evidenciado que una infección viral, en este caso en particular, puede ser el condicionante de un $\mathrm{ACV}$ isquémico agudo, relacionado con el efecto protrombótico de la respuesta inflamatoria ${ }^{(19)}$. Sin embargo, en nuestro paciente, hasta antes del evento motivo de ingreso, era un paciente aparentemente sano, sin ningún síntoma evidente de alguna enfermedad, tal como lo expresan sus exámenes de laboratorio, al ingreso, tanto el hemograma como el bioquímico. Además, incidentalmente se evidencia que el $\mathrm{DD}$, se encontraba en valores relativamente altos y con plaquetas normales.

En el estudio retrospectivo de $\mathrm{Li}{ }^{(12)}$, en febrero del presente año, demuestra que de 221 pacientes confirmados con COVID-19, 13 pacientes (5,9\%) llegaron a presentar enfermedad cerebrovascular después de la infección por SARS-CoV-2. De estos pacientes, A 11 (84,6\%) se les diagnostica con $A C V$ isquémico, 1 paciente $(7,7 \%)$ con trombosis de seno venoso cerebral y por último, 1 paciente $(7,7 \%)$ con hemorragia cerebral. La edad de estos pacientes, presentaba el rango entre los 57 a 91 años de edad con una media de 73,5 años. Se destaca que 11 de los 13 pacientes presentaban infección por COVID-19 severo; lo que sugiere ser indicador de ACV isquémico ${ }^{(12)}$.

También se tiene el reporte de un paciente de 79 años que el pasado 24 de enero del 2020 fue reportado en el Hospital de Medicina Tradicional China. En el mencionado caso, el paciente presentó la sintomatología de un ACV en curso y, más tarde, se demostró la presencia del SARSCoV-2, por lo que se le relacionó con el cuadro clínico, de forma muy parecida a nuestro paciente, motivo del reporte. En este caso reportado se describe que el COVID-19, causó hipoxemia y una tormenta excesiva de citoquinas inflamatorias, que contribuyen a la aparición y desarrollo de accidente cerebrovascular isquémico, por la producción de micro trombos en forma masiva. Los pacientes adultos mayores presentan factores de riesgo más elevados con $\mathrm{ACV}$ isquémicos y eventos vasculares embolico altos ${ }^{(20)}$. Sin embargo, no es una regla en el diagnóstico, puesto que el paciente de estudio, quien es relativamente joven, con 40 años, sin ningún antecedente de enfermedad previa, presenta sin embargo en forma insidiosa, progresiva y rápida, toda la sintomatología probablemente planteada como normal para una persona con casi el doble de su edad.

Por lo tanto, con este tipo de pacientes y las complicaciones que se presentan, se abre una nueva arista en el manejo, tratamiento y pronóstico, por lo que los neurólogos deben de prepararse para la batalla contra la COVID-19, en especial cuando el conocimiento de la enfermedad sigue siendo limitada. Se concluye que la injuria cerebral aguda ha sido descrita en medio de la pandemia por SARS-CoV-2 como parte de las manifestaciones neurológicas, y dentro de estas, los infartos cerebrales por oclusión de grandes vasos, lo que 
agravaría el pronóstico del paciente, independientemente de la presencia de sintomatología respiratoria clásica en la presentación por infección de COVID-19. Más aun teniendo en cuenta que esta se presenta independientemente de la edad y de factores de riesgo preexistentes.

\section{REFERENCIAS BIBLIOGRÁFICAS}

1. Antipova D, Eadie L, Macaden A, Wilson P. Diagnostic accuracy of clinical tools for assessment of acute stroke: a systematic review. BMC Emerg Med. 2019 Sep 4; 19(1): 49. doi: http://doi.org/10.1186/s12873-019-0262-1

2. Casaubon LK, Boulanger JM, Glasser E, Blacquiere D, Boucher S, Brown K, et al. Canadian Stroke Best Practice Recommendations: Acute Inpatient Stroke Care Guidelines, Update 2015. Int J Stroke 2016; 11(2): 23952. doi: http://doi.org/10.1177/1747493015622461

3. Cumbler E, Wald H, Bhatt DL, Cox M, Xian Y, Reeves $M$, et al. Quality of care and outcomes for in-hospital ischemic stroke: findings from the National Get With The Guidelines-Stroke. Stroke 2014; 45(1): 231-8. doi: http:// doi.org/10.1161/STROKEAHA.113.003617.

4. World Stroke Organization (WSO). Stroke Guideline Development Handbook 2009 [Internet] [Consultado julio 2018] Disponible en: http://www.world-stroke.org.

5. Wang Y, Chen Y, Qin Q. Unique epidemiological and clinical features of the emerging 2019 novel coronavirus pneumonia (COVID-19) implicate special control measures [published online ahead of print, 2020 Mar 5]. J Med Virol. 2020; 92(6): 568-576. doi: http://doi. org/10.1002/jmv.25748.

6. Guo T, Fan $Y$, Chen $M$, Wu X, Zhang $L$, He T, et al. Cardiovascular Implications of Fatal Outcomes of Patients With Coronavirus Disease 2019 (COVID-19). JAMA Cardiology. 2020 Mar 27. doi: http://doi. org/10.1001/jamacardio.2020.1017.

7. Zhao K, Huang J, Dai D, Feng Y, Liu L, Nie S. Acute myelitis after SARS-CoV-2 infection: a case report. medRxiv preprint. 2020 Abr 9. doi: https://doi. org/10.1101/2020.03.16.20035105

8. Mao L, Wang M, Chen S, He Q, Chang J, Hong C, et al. Neurological manifestations of hospitalized patients with COVID-19 in Wuhan, China: a retrospective case series study. SSRN Journal. 2020 Feb 25. doi: https://doi. org/10.1101/2020.02.22.20026500

9. GenBank. Wuhan seafood market pneumonia virus isolate Wuhan-Hu-1, complete genome [Internet] [Consultado 2020 Ene 23] Disponible en: http://www. ncbi.nlm.nih.gov/nuccore/MN908947.3.
10. Li YC, Bai WZ, Hashikawa T. The neuroinvasive potential of SARS-CoV2 may play a role in the respiratory failure of COVID-19 patients. J Med Virol. 2020; 92(6): 552-555. doi: http://doi.org/10.1002/jmv.25728.

11. Lyden P. Temporary Emergency Guidance to US Stroke Centers During the Coronavirus Disease 2019 (COVID-19) Pandemic. Stroke. 2020; 51(6): 1910-1912. http://doi.org/10.1161/STROKEAHA.120.030023.

12. Li $Y$, Wang $M$, Zhou $Y$, Chang J, Xian $Y$, Mao L, et al. Acute Cerebrovascular Disease Following COVID-19: A Single center, Retrospective, Observational Study (3/3/2020). 2020. Available at http://dx.doi.org/10.2139/ ssrn.3550025

13. Guo T, Fan $Y$, Chen $M$, Wu $X$, Zhang $L$, He $T$, et al. Cardiovascular Implications of Fatal Outcomes of Patients With Coronavirus Disease 2019 (COVID-19). JAMA Cardiology. 2020. Mar 27 doi: http://doi. org/10.1001/jamacardio.2020.1017

14. Hai-Yang W, Xue-Lin L, Zhong-Rui Y, Xiao-Pei S, Jie $\mathrm{H}$, Bing-Wei Z. Potential neurological symptoms of COVID-19. Ther Adv Neurol Disord 2020 Mar 28; 13: 1-2. https://doi.org/10.1177/1756286420917830.

15. Poyiadji N, Shahin G, Noujaim D, Stone M, Patel S, Griffith B. COVID-19-associated Acute Hemorrhagic Necrotizing Encephalopathy: CT and MRI Features. Radiology. 2020 Mar 31. doi: http://doi.org/10.1148/radiol.2020201187.

16. Xu Z, Shi L, Wang Y, Zhang J, Huang L, Zhang C, et al. Pathological findings of COVID-19 associated with acute respiratory distress syndrome. Lancet Respir Med. 2020 Feb 17; 8: 420-422. doi: https://doi.org/10.1016/S22132600(20)30076-X.

17. Wu Y, Xu X, Chen Z, Duan J, Hashimoto K, Yang L, et al. Nervous system involvement after infection with COVID-19 and other coronaviruses. Brain, Behavior, and Immunity. 2020 Mar 30. doi: https://doi.org/10.1016/j. bbi.2020.03.031.

18. Wang $Y$, Chen $Y$, Qin Q. Unique epidemiological and clinical features of the emerging 2019 novel coronavirus pneumonia (COVID-19) implicate special control measures. J Med Virol. 2020; 92(6): 568-576. doi: http:// doi.org/10.1002/jmv.25748.

19. Grau AJ, Buggle F, Becher H, Zimmermann E, Spiel M, Fent $T$, et al. Recent bacterial and viral infection is a risk factor for cerebro vascular ischemia: clinical and biochemical studies. Neurology. 1998 Ene; 50(1): 196203. doi: 10.1212/wnl.50.1.196.

20. Zhai P, Ding Y, Li Y. The impact of COVID-19 on ischemic stroke: A case report. Research Square. 2020 Mar 31. doi: http://doi.org/10.21203/rs.3.rs-20393/v1 